\title{
Magnetic and Langmuir Probe Measurements on the Plasmoid Thruster Experiment (PTX)
}

\author{
Syri J. Koelfgen" \\ University of Alabama in Huntsville (UAH), Propulsion Research Center, S225 Technology Hall, \\ Huntsville, AL 35899 \\ Richard Eskridge, ${ }^{\dagger}$ Michael H. Lee, ${ }^{\ddagger}$ and Adam Martin ${ }^{8}$ \\ NASA Marshall Space Flight Center (MSFC), Propulsion Research Center TD40, Huntsville, AL 35812 \\ Clark W. Hawk,' and Peter Fimognari" \\ University of Alabama in Huntsville (UAH), Propulsion Research Center, S225 Technology Hall, \\ Huntsville, AL 35899
}

The Plasmoid Thruster Experiment (PTX) operates by inductively producing plasmoids in a conical theta-pinch coil and ejecting them at high velocity. A plasmoid is a plasma with an imbedded closed magnetic field structure. The shape and magnetic field structure of the translating plasmoids have been measured with of an array of magnetic field probes. Six sets of two B-dot probes were constructed for measuring $B_{z}$ and $B_{0}$, the arial and azimuthal components of the magnetic field. The probes are wound on a square $\mathbf{G - 1 0}$ form, and have an average (calibrated) $N A$ of $9.37 \times 10^{-5} \mathrm{~m}^{2}$, where $N$ is the number of turns and $A$ is the cross-sectional area. The probes were calibrated with a Helmholtz coil, driven by a highvoltage pulser to measure $N A$, and by a signal generator to determine the probe's frequency response. The plasmoid electron number density $n_{e}$ electron temperature $T_{e}$ and velocity ratio $v / c_{m}$ (where $v$ is the bulk plasma flow velocity and $c_{m}$ is the ion thermal speed) have also been measured with a quadruple Langmuir probe. The Langmuir probe tips are $10 \mathrm{~mm}$ long, 20-mil diameter stainless steel wire, housed in a 6-inch long 4-bore alumina rod. Measurements on PTX with argon and hydrogen from the magnetic field probes and quadruple Langmuir probe will be presented in this paper.

\section{Nomenclature}

$A=$ cross-sectional area of $\mathrm{B}$-dot probes

$A_{1}, A_{2}, A_{3}, A_{4}=$ collection area of quadruple Langmuir probe electrodes $1,2,3$, and 4

$B, \mathrm{~B} \quad=$ magnetic field

$B_{z} \quad=$ axial component of magnetic field

$B_{\theta} \quad=$ azimuthal component of magnetic field

c. $\quad=$ ion thermal speed

$e_{c} \quad=$ electron charge

$e e \lambda_{\text {mfp }} \quad=$ electron-electron collision mean free path

ii $\lambda_{\text {mpp }}=$ ion-ion collision mean free path

$I_{1}, I_{2}, I_{3}, I_{4}=$ current collected by quadruple Langmuir probe electrodes $1,2,3$, and 4

$I_{B} \quad=$ Bohm current

$I_{i} \quad=$ current collected at Langmuir probe tip

\footnotetext{
- NASA/MSFC GSRP Fellow and UAH Graduate Research Assistant, AIAA Student Member.

${ }^{\dagger}$ NASA/MSFC Aerospace Systems Engineer, AIAA Member.

INASA/MSFC Electrical Engineer.

${ }^{5}$ NASA/MSFC Physicist.

IUAH Propulsion Research Center Director and Mechanical \& Aerospace Engineering Professor, AIAA Fellow.

" UAH Graduate Research Assistant.
} 


$\begin{array}{ll}\mathbf{J} & =\text { current density } \\ J_{c} & =\text { electron saturation current density } \\ J_{i} & =\text { ion saturation current density } \\ L & =\text { Langmuir probe electrode length } \\ \lambda_{D} & =\text { Debye length } \\ m_{e} & =\text { electron mass } \\ M_{i} & =\text { ion mass } \\ n_{e} & =\text { electron number density } \\ N & =\text { number of turns of B-dot probe } \\ r_{p} & =\text { Langmuir probe electrode radius } \\ s & =\text { clearance between Langmuir probe electrodes } \\ t & =\text { time } \\ t_{0} & =\text { initial time } \\ T_{e} & =\text { electron temperature in eV } \\ v & =\text { bulk plasma velocity } \\ V_{B o h m} & =\text { Bohm velocity } \\ V & =\text { voltage induced in B-dot probe coil } \\ V_{1}, V_{2}, V_{3}, V_{4} & =\text { potential of Langmuir probe electrodes } 1,2,3 \text { and } 4 \\ V_{d 2} & =\text { potential difference between probes } 2 \text { and } 1 \\ V_{d B} & =\text { applied potential difference between probes } 3 \text { and } 1 \\ V_{d 4} & =\text { applied potential difference between probes } 4 \text { and } 1 \\ V_{o w} & =\text { B-dot probe passive integrator output signal } \\ V_{p} & =\text { plasma potential } \\ & \end{array}$

\section{Introduction}

THE plasmoid thruster is a pulsed inductive rocket that utilizes the $\mathbf{J}$ × $\mathbf{B}$ (Lorentz) force to accelerate plasmoids and produce thrust (Fig. 1). A plasmoid is a plasma with an internal magnetic field structure, also known as a compact toroid.' Plasmoid accelerators have been used for fueling fusion devices ${ }^{2-4}$ and their application to space propulsion has been proposed and studied theoretically. ${ }^{5-7}$ The Plasmoid Thruster Experiment (PTX) is an experiment to evaluate the use of plasmoids for propulsion. ${ }^{8}$ The plasmoids are formed inductively in a conical theta-pinch coil, which eliminates the problems associated with discharging a large current across electrodes to produce and accelerate the plasma. Electrode erosion is a limiting factor in the functional lifetime of many pulsed electromagnetic accelerators, such as the magnetoplasmadynamic (MPD) thruster., 5,9 The plasmoid thruster is an option for removing this limitation. Additionally, since the plasmoid has a self-contained magnetic field and is therefore not connected to any external magnetic field lines, the problem of detachment is reduced. Magnetic insulation of the plasma should also lead to reduced thermal losses to the walls. An actual plasmoid thruster would operate repetitively at a frequency of $10-100 \mathrm{~Hz}$ and would use solid-state switching. In the present experiment, only a single plasmoid is formed and accelerated;

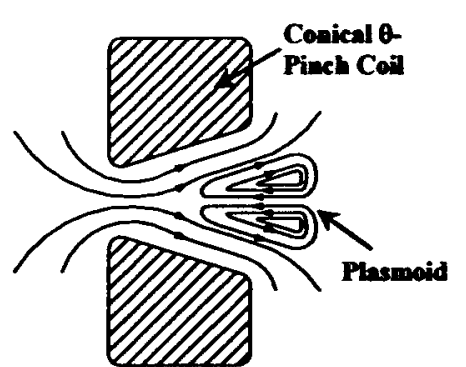

Figure 1. Plasmoid in Conical O-Pinch Coil PTX is not yet optimized.

\section{PTX}

In PTX, the plasmoids are produced in a Pyrex tube, which serves as the vacuum chamber, situated inside of a single-tum conical theta-pinch coil with a half-angle of $17.5^{\circ}$ (Fig. 2). The Pyrex tube is connected to a rectangular vacuum chamber for diagnosis of the exhaust plume. The theta-pinch coil is driven by a $560 \mathrm{nF}, 40 \mathrm{kV}$ capacitor bank that is switched by a Perkin-Elmer GP-32B spark-gap switch. The coil and capacitor bank constitute a tank (LC) circuit that rings sinusoidally with a peak current of $53 \mathrm{kA}$ at $35 \mathrm{kV}$ charge voltage (Fig. 3). The bank is fired by sending an optical trigger signal to a high-voltage pulser that then triggers the spark-gap switch. When triggered, the spark-gap closes, allowing the capacitor bank to discharge through the theta-pinch coil. A solenoid valve injects propellant into the chamber, before the bank is discharged at a time $t_{0}$. (The time before the bank discharge at which 
propellant is injected is referred to as the puff valve delay.) The rapidly changing axial magnetic field creates an azimuthal electric field that preionizes the plasma. Simultaneously, the plasma is seeded with the magnetic field produced during the first half-cycle of the sinusoidal discharge. When the current swings through zero, the field is reversed and the chamber contains a cold, partially ionized plasma with an imbedded magnetic field. As the external magnetic field increases in the opposite direction, it reconnects with the bias field and the plasma is compressed and fully ionized.' During the formation process, the interaction of the magnetic field with the plasma induces a large azimuthal current in the plasma. The resultant $\mathrm{J} \times \mathrm{B}$ force accelerates the plasmoid away from the coil and generates thrust. Argon and hydrogen gases were chosen to model heavyweight and lightweight propellant in PTX.

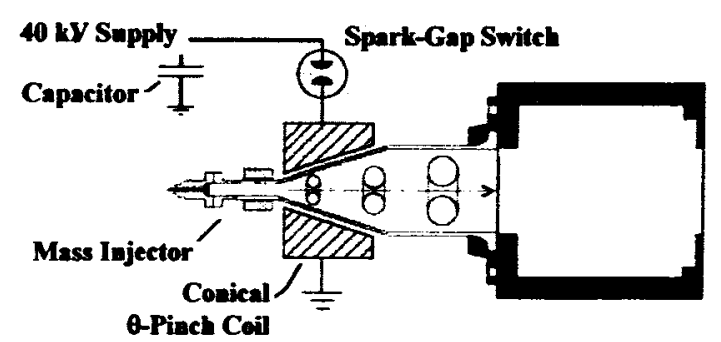

Figure 2. Plasmoid Thruster Experiment

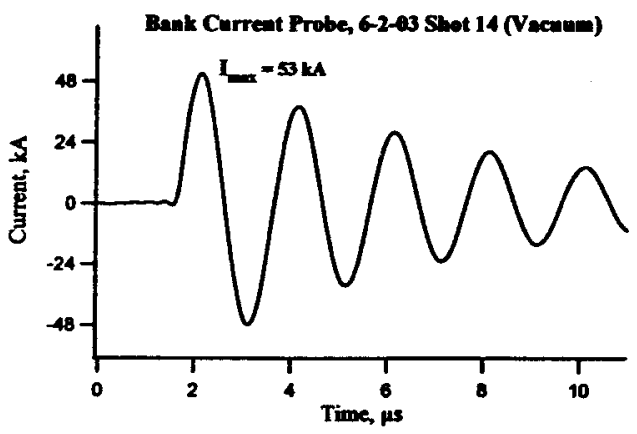

Figure 3. PTX Capacitor Bank Discharge

\section{Diagnostics}

A variety of diagnostics are used on PTX, including:

a. a high-speed Cordin $220 \mathrm{~B}$ framing camera (with eight independently triggered CCD arrays; inter-frame time as low as $10 \mathrm{~ns}$ ), for imaging and estimating the plasmoid velocity;

b. an excluded flux array (an array of magnetic field probes and flux loops), for determining the shape of the plasmoid in the coil;

c. a light pipe velocimeter (fiber optic cables fed into fast PIN photo diodes), for measuring velocity;

d. a heterodyne, quadrature HeNe laser intefferometer, for measuring the line-averaged electron density, $n_{i}$

e. an array of magnetic field sensors (B-dot probes), for measuring axial and azimuthal magnetic field; and

f. a quadruple Langmuir probe, for measuring the plasmoid electron temperature $T_{e}$ number density $n_{\infty}$ and velocity ratio $v / c_{m}$, where $v$ is the bulk plasma flow velocity and $c_{m}$ is the ion thermal speed.

This paper will focus of the B-dot probe array and quadruple Langmuir probe.

\section{A. Internal B-dot Probe Array}

An array of internal B-dot probes ${ }^{10,11}$ has been constructed and installed in the exhaust chamber, perpendicular to the z-axis (Fig. 4). Six sets of two B-dot probes measure the axial and azimuthal magnetic fields ( $B_{z}$ and $B_{\theta}$ ) of the plasmoid as it translates past the probe array. The probes were wound with 36 gauge magnet wire on a $3.15 \mathrm{~mm}$ square G-10 form (Fig. 4). The probe signals are passively integrated $(\mathrm{RC} \approx 20 \mu \mathrm{s})$ for improved dynamic range. The output signals from the passive integrator, $V_{\text {own }}$ are recorded with Acqiris digitizers (8-bit, $250 \mathrm{Msamples} / \mathrm{s}$ ). The magnetic field measured by each probe is given by:

$$
B=\int \dot{B} d t \equiv \frac{1}{N A} \int V d t=\left(\frac{1}{N A}\right)\left(R C V_{\text {out }}+\int V_{\text {out }} d t\right)
$$




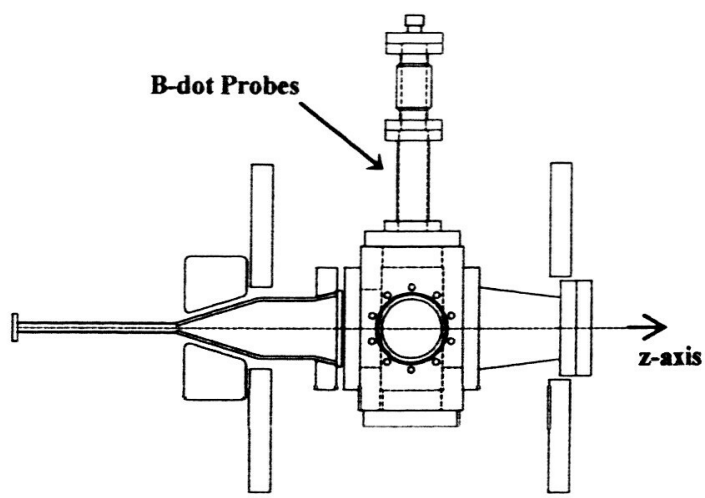

a) Internal B-dot Probe Array on PTX

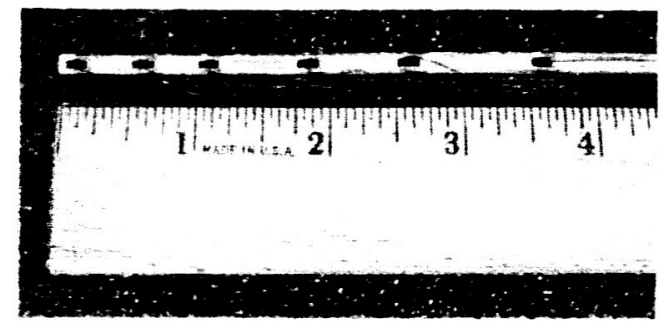

b) Internal B-dot Probe Array

\section{Figure 4. Internal B-dot Probe Array}

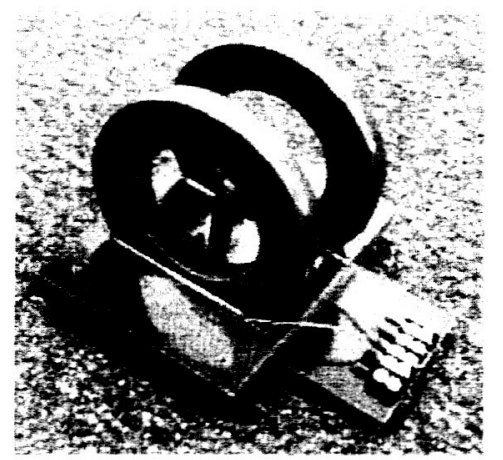

Figure 5. Helmholtz Coil

The B-dot probes were calibrated with a custom-built Helmholtz coil (Fig. 5). The Helmholtz coil is a set of two coaxial current loops with equal radii, separated from each other by a distance equal to that radius. The radius and separation of the loops is approximately 2.5 inches. Equal amounts of current flowing in the same direction are applied to the loops, producing an extraordinarily uniform magnetic field inside the volume of the Helmholtz coil. The PTX Helmholtz coil was calibrated with a DC power supply, multimeter, and Hall probe to determine the magnetic field inside the Helmholtz coil as a function of applied current.

To calibrate the individual B-dot probes in the array, the Helmholtz coil was driven with a 300 Volt pulser, and the resulting voltage responses of the Helmholtz coil and each B-dot probe were recorded with a digital oscilloscope. The calibrated quantity, $N A$, of the probes was found to be within $20 \%$ of the design value of $N A=10 \times(3.15 \mathrm{~mm})^{2}=9.92 \times 10^{-5} \mathrm{~m}^{2}$. The frequency response of the B-dot probes was also assessed by applying a sine wave to the Helmholtz coil at frequencies ranging from $1 \mathrm{kHz}$ to $12 \mathrm{MHz}$. The resulting Helmholtz coil signal (obtained with a Pearson current probe) and the B-dot probe responses were measured with the oscilloscope and combined to determine $N A$ as a function of frequency.

\section{B. Quadruple Langmuir Probe}

A quadruple Langmuir probe ${ }^{12}$ has also been constructed (Fig. 6) to make local measurements of the plasmoid electron temperature $T_{e}$, electron number density $n_{e}$, and velocity ratio $v / c_{m}$. Three of the four probes are aligned parallel to the plasma flow (the $\mathrm{z}$-axis); the fourth probe is aligned perpendicular. The Langmuir probe tips are $10 \mathrm{~mm}$ long, $0.5 \mathrm{~mm}$ (20 mil) diameter stainless steel wires, housed in a $152 \mathrm{~mm}$ long, 4-bore alumina rod. The alumina rod is torr-sealed into a stainless steel tube and installed at the downstream end of the PTX exhaust chamber through a sliding O-ring seal, so that measurements may be taken along the $\mathrm{z}$-axis.

An electrical schematic of the quadruple Langmuir probe is shown in Fig. 7. Probe 2 is unbiased, and so it floats to a potential such that it does not collect any current. Probes 3 and 4 are biased at approximately equal voltages $\left(V_{d 3}\right.$ and $\left.V_{d 4}\right)$ relative to probe 1.

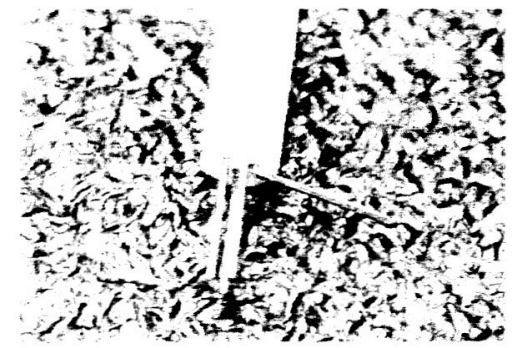

Figure 6. Quadruple Langmuir Probe

Probe 1 is biased above the floating potential and below electron saturation, and emits current. Probes 3 and 4 are biased in the ion saturation region of the ideal probe characteristic, and collect current. For both probes 3 and 4 , the 


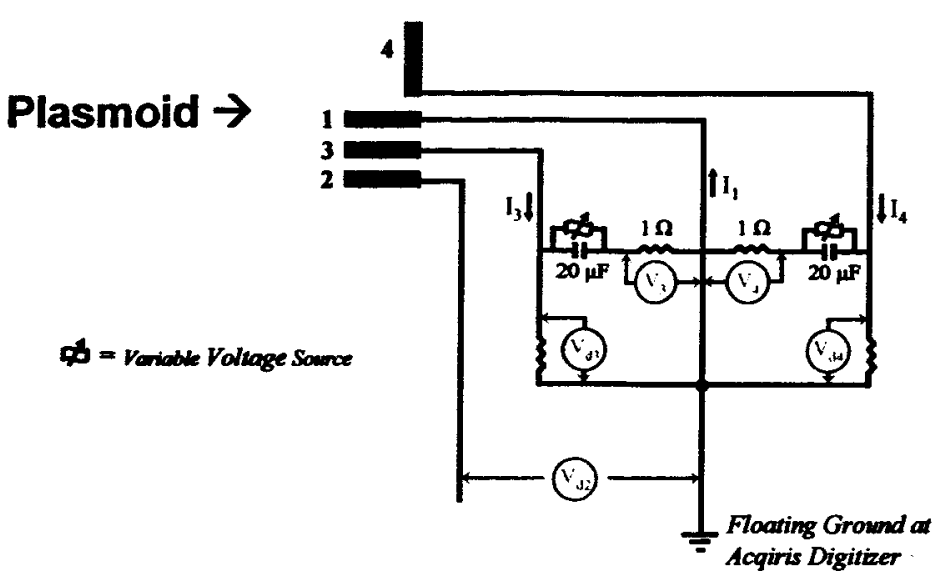

bias voltage is produced by a variable DC power supply, with $20 \mu \mathrm{F}$ of capacitance in parallel, in order to source the current on the fast time scale of the experiment.

Quadruple Langmuir probe theory ${ }^{12,13}$ is a combination of triple probe theory ${ }^{14,15}$ and a crossed electrostatic probe technique. ${ }^{16,17}$ The measured quantities for data analysis are the currents collected by probes 3 and $4\left(I_{3}\right.$ and $\left.I_{4}\right)$, and the potential differences of probes 2,3 , and 4 relative to probe 1 , called $V_{d Q}, V_{d B}$, and $V_{d 4}$ respectively, where

Figure 7. Quadruple Langmuir Probe Cireuit

$$
V_{d 2}=V_{2}-V_{1}
$$

and

$$
\begin{aligned}
& V_{d 3}=V_{3}-V_{1} \\
& V_{d 4}=V_{4}-V_{1} . \\
& V_{d 3}=V_{d 4}
\end{aligned}
$$

Simultaneous collection of $I_{3}, I_{4}, V_{d}, V_{A B}$, and $V_{d 4}$ yield measurements of $T_{e}, n_{e}$, and $v / c_{m}$. Analysis of the probe response assumes that several conditions are met, including:

a. the probe is operating in the collisionless sheath regime, i.e. the condition $\frac{e e \lambda_{\operatorname{mfp}}}{\lambda_{D}} \gg 1$ is met, where $e e \lambda_{\text {mpp }}$ is the electron-electron collision mean free path and $\lambda_{D}$ is the Debye length;

b. the sheath around each probe electrode is thin, as prescribed by $\frac{r_{p}}{\lambda_{D}} \gg 1$, where $r_{p}$ is the Langmuir probe electrode radius;

c. the probe is in the free molecular regime, so the effect of free-streaming particle collisions with the probe can be ignored, such that $\frac{e e \lambda_{\operatorname{mpp}}}{r_{p}}>1$ and $\frac{i i \lambda_{\operatorname{mgp}}}{r_{p}}>1$ are satisfied, where $i i \lambda_{\text {mpp }}$ is the ion-ion collision mean free path;

d. the end effect is minimized, which is achieved if the plasma velocity $v$ (the heavy-particle velocity) is small compared to the Bohm velocity $v_{B o h m}$, so that there is not a large number of ions reaching the probe causing a peak in the measured ion current, such that the condition $L>50 \lambda_{D} \frac{v}{v_{B o h m}}$ is satisfied, where $L$ is the length of the probe tip; 
e. the error introduced to Langmuir probe current measurements by probe tip currents is less than approximately $10 \%$, i.e. $\frac{I_{1}}{I_{B}}=\frac{1}{2} \frac{r_{p}}{L} \frac{v}{v_{B o l m}} \leq 0.1$; and

f. the clearance $s$ between probe electrodes is sufficient so that sheath interactions between adjacent electrodes is avoided, such that $\frac{s}{\lambda_{D}}>250$.

The PTX quadruple Langmuir probe was designed to ensure that these conditions were adequately met under expected operating conditions.

\section{Electron Temperature}

This paper takes a slightly different approach to the derivations of the formulas for $T_{e}$ and $n_{e}$, however the results are equivalent to those found in other works. ${ }^{12,13}$ To determine an expression for the electron temperature from the quadruple Langmuir probe measurements, we begin with equations describing the current collected by probes 1,2 , and 3:

$$
\begin{aligned}
& I_{1}=J_{e} A_{1} \exp \left(\frac{V_{1}-V_{p}}{T_{e}}\right)-J_{i} A_{1} \\
& I_{2}=J_{e} A_{2} \exp \left(\frac{V_{2}-V_{p}}{T_{e}}\right)-J_{i} A_{2} \\
& -I_{3}=J_{e} A_{3} \exp \left(\frac{V_{3}-V_{p}}{T_{e}}\right)-J_{i} A_{3}
\end{aligned}
$$

where $A_{1}, A_{2}$, and $A_{3}$ are the current collection areas of probes 1,2 , and $3, V_{p}$ is the plasma potential, $T_{e}$ is the electron temperature in units of electron volts $(\mathrm{eV})$, and $J_{e}$ and $J_{i}$ are the electron and ion saturation current densities, as given by:

$$
\begin{gathered}
J_{e}=n_{e} e_{c}^{3 / 2} \sqrt{\frac{T_{e}}{2 \pi m_{e}}} \\
J_{i}=\exp \left(-\frac{1}{2}\right) n_{e} e_{c}^{3 / 2} \sqrt{\frac{T_{e}}{M_{i}}}
\end{gathered}
$$

where $n_{e}$ is the electron number density, $e_{c}$ is the electron charge, $m_{e}$ is the electron mass, and $M_{i}$ is the ion mass. Since the current emitted by probe 1 is collected by both probes 3 and 4 , and since $I_{1}$ flows in the opposite direction of $I_{3}$ and $I_{4}$ (as shown in Fig. 7),

$$
I_{1}=-\left(I_{3}+I_{4}\right)=-I_{3}\left(1+\frac{I_{4}}{I_{3}}\right)
$$


Given that $A_{1}=A_{2}=A_{3}$, and substituting Eq. (9) into Eq. (4), we have:

$$
I_{1}=-J_{e} A_{3}\left[\exp \left(\frac{V_{3}-V_{p}}{T_{e}}\right)-\frac{J_{i}}{J_{e}}\right]\left(1+\frac{I_{4}}{I_{3}}\right)
$$

Since probe 2 is floating and therefore $I_{2}=0$, Eq. (5) reduces to

$$
\frac{J_{i}}{J_{e}}=\exp \left(\frac{V_{2}-V_{p}}{T_{e}}\right)
$$

Equating Eq. (4) and Eq. (10), noting that $A_{1}=A_{3}$, dividing by $J_{e}$, and applying Eq. (11) yields:

$$
\exp \left(\frac{V_{1}-V_{p}}{T_{e}}\right)-\exp \left(\frac{V_{2}-V_{p}}{T_{e}}\right)=-\left(1+\frac{I_{4}}{I_{3}}\right)\left[\exp \left(\frac{V_{3}-V_{p}}{T_{e}}\right)-\exp \left(\frac{V_{2}-V_{p}}{T_{e}}\right)\right]
$$

Multiplying Eq. (12) by $-\exp \left(\frac{V_{p}}{T_{e}}\right)$ and $\exp \left(-\frac{V_{1}}{T_{e}}\right)$, and applying Eq. (2) and Eq. (3a), we are left with an expression for $T_{c}$ (in $\mathrm{eV}$ ) as a function of the measured quantities $I_{3}, I_{4}$, and $V_{\mathcal{A}}$ as well as the applied voltage $V_{\mathcal{A}}$ :

$$
\exp \left(\frac{V_{d 2}}{T_{e}}\right)-1=\left(1+\frac{I_{4}}{I_{3}}\right)\left[\exp \left(\frac{V_{d 3}}{T_{e}}\right)-\exp \left(\frac{V_{d 2}}{T_{e}}\right)\right]
$$

2. Electron Density

To derive an expression for the electron density, Eq. (6) is represented as:

$$
-I_{3}=J_{e} A_{3} \exp \left(\frac{V_{3}}{T_{e}}\right) \exp \left(\frac{-V_{p}}{T_{e}}\right)-J_{i} A_{3} .
$$

Rearranging Eq. (11) into

$$
\exp \left(\frac{-V_{p}}{T_{e}}\right)=\frac{J_{i}}{J_{e}} \exp \left(\frac{-V_{2}}{T_{e}}\right)
$$

substituting this into Eq. (14), and using Eq. (2) and Eq. (3a), we are left with: 


$$
-I_{3}=J_{i} A_{3}\left[\exp \left(\frac{V_{d 3}-V_{d 2}}{T_{e}}\right)-1\right]
$$

Substituting Eq. (8) into Eq. (16), and rearranging, yields the expression for $n_{e}$ :

$$
n_{e}=\frac{-I_{3}}{\exp \left(-\frac{1}{2}\right) e_{c}^{3 / 2} A_{3} \sqrt{\frac{T_{e}}{M_{i}}}\left[\exp \left(\frac{V_{d 3}-V_{d 2}}{T_{e}}\right)-1\right]}
$$

\section{Velocity}

In addition to measurements of $T_{e}$ and $n_{e}$, the quadruple Langmuir probe also provides a measurement of the velocity ratio $v / c_{m}$ from the collected currents $I_{3}$ and $I_{4}$. For $V_{d B}=V_{d 4}$, with probe 3 aligned with the flow vector and probe 4 perpendicular to it, the ratio of $I_{3}$ to $I_{4}$ has been shown to be $e^{17}$

$$
\frac{I_{4}}{I_{3}}=\frac{2 A_{4}}{\sqrt{\pi} A_{3}} \exp \left[-\left(\frac{v}{c_{m}}\right)^{2}\right] \sum_{n=0}^{\infty}\left[\frac{\left(\frac{v}{c_{m}}\right)^{n}}{n !}\right]^{2} \Gamma\left(n+\frac{3}{2}\right)
$$

The velocity ratio $v / c_{m}$ is obtained by iteration of Eq. (19).

\section{Experimental Results}

The B-dot probe array is mounted on the top of the PTX exhaust chamber, so the plasmoid magnetic field can be measured at various vertical positions in the chamber (Fig. 8). In Fig. 8(a), probe B_z3, the third axial B-dot probe in the array, is positioned on the centerline of the theta-pinch coil and chamber. Probe B_zl is in the lowest vertical position (below the centerline) and B_z6 is in the highest vertical position. In Fig. 8(b), probe $B_{-}-5$ is on the centerline.

For the same operating conditions (argon at 6.5 psig manifold pressure injected at a $2600 \mu$ s puff valve delay), PTX was nun with the B-dot probe array in the two different vertical positions on two different days. This was done so that the plasmoid magnetic fields could be mapped

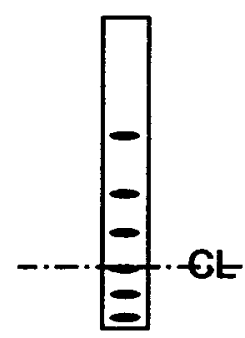

(a) Probe B_z3 on CL $\quad$ (b) Probe $\mathrm{B} \_55$ on CL

Figure 8. B-dot Probe Array Positions in Relation to the PTX Centerline (CL)

over the entire vertical cross-section of the chamber. On February 23, 2004 Shot3, the B-dot probe array positioning shown in Fig. 8(a) was used, and on February 25, 2004 Shot 43, the configuration in Fig. 8(b) was used. The axial and azimuthal magnetic fields as a function of time were obtained for both shots. The magnetic field averaged over several data points about $\mathbf{1 8 . 6 5} \mu$ s (the time near the axial magnetic field peak) was then determined for each B-dot probe, so that the magnetic field could be graphed as a function of vertical position, as each B-dot probe corresponds to a different vertical position in the chamber.

The axial magnetic field profile vs. vertical position for the two shots at $18.65 \mu$ s is shown in Fig. 9 (a). A Cordin camera photo of the plasmoid translating toward the B-dot probe array is shown in Fig. $9(\mathrm{~b})$. The light pipe 
velocimeter measured plasmoid velocities of $28 \mathrm{~km} / \mathrm{s}$ on February 23, 2004 Shot 3 and $22 \mathrm{~km} / \mathrm{s}$ on February 25 , 2004 Shot 43. For the same operating conditions, the quadruple Langmuir probe was also used to measure $T_{e}, n_{e}$, and $v / c_{m}$ as a function of time (Fig. 10).

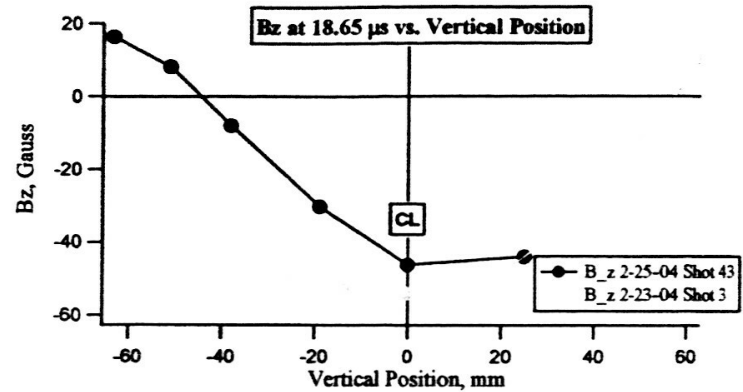

(a) Bz vs. Vertical Position

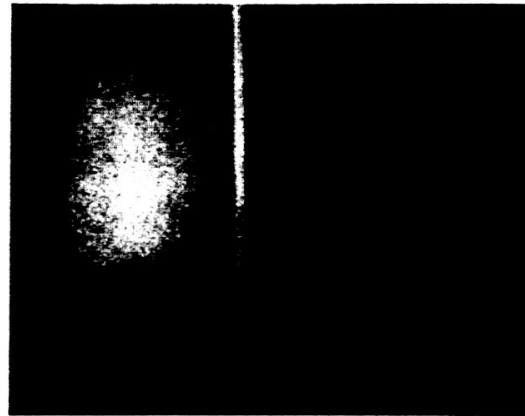

(b) Cordin Photo from 2-25-04 Shot 43

Figure 9. Plasmoid Magnetic Field vs. Vertical Position and Cordin Camera Photograph, 6.5 psig Argon at $2600 \mu$ s delay
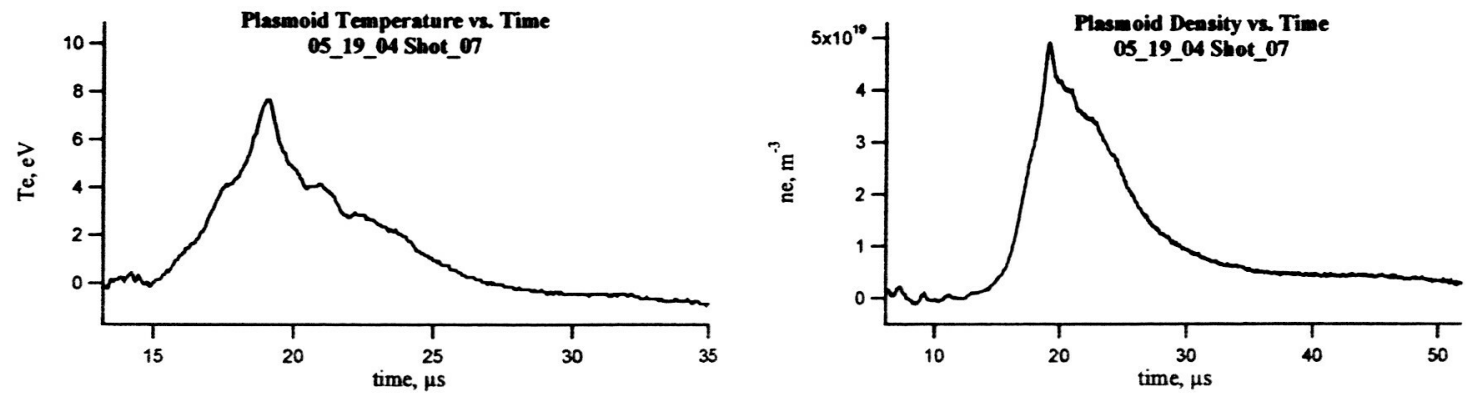

Figure 10. Plasmoid Temperature and Density, May 19, 2004, Shot 7, 6.5 psig Argon at $2600 \mu$ delay

Figure 9(a) is consistent with the axial magnetic fields expected in a plasmoid. Figure 10 shows a maximum electron temperature of $7.6 \mathrm{eV}$ and a maximum electron number density of $4.9 \times 10^{19} \mathrm{~m}^{-3}$. At $18.65 \mu \mathrm{s}$, the temperature is $6.3 \mathrm{eV}$ and the density is $4.0 \times 10^{19} \mathrm{~m}^{-3}$. In determining the velocity ratio $v / c_{m}$, the summation term in Eq. (19) reaches a constant value after 50 terms, so for $n=0 . .50$, the peak velocity ratio for this test was approximately 3.2 .

For comparison to argon, hydrogen was used as a lightweight propellant. The axial and azimuthal magnetic fields in PTX for hydrogen at 38.6 psig manifold pressure, injected $2400 \mu$ s before the bank discharge, are shown in Fig. 11. Numbers corresponding to each B-dot probe are placed on their corresponding curve, where " 1 " denotes the magnetic field curve obtained from B-dot probe $\mathrm{zl}$ or $\theta 1$. In Fig. 11 we see that the azimuthal magnetic field, $\mathrm{B} \theta$, reverses direction, which is consistent with the field-reversal characteristic of plasmoids. The noise in the beginning of the magnetic field plots is pick-up from the discharge of the capacitor bank. Figure 12 shows the electron temperature and density profiles for similar operating conditions. The temperatures at the first and second peaks are $22 \mathrm{eV}$ and $23 \mathrm{eV}$. The corresponding densities at the two peaks are $1.1 \times 10^{20} \mathrm{~m}^{-3}$ and $1.2 \times 10^{20} \mathrm{~m}^{-3}$. The velocity ration $v / c_{m}$ on the first peak in Fig. 12 is approximately 0.4 . 

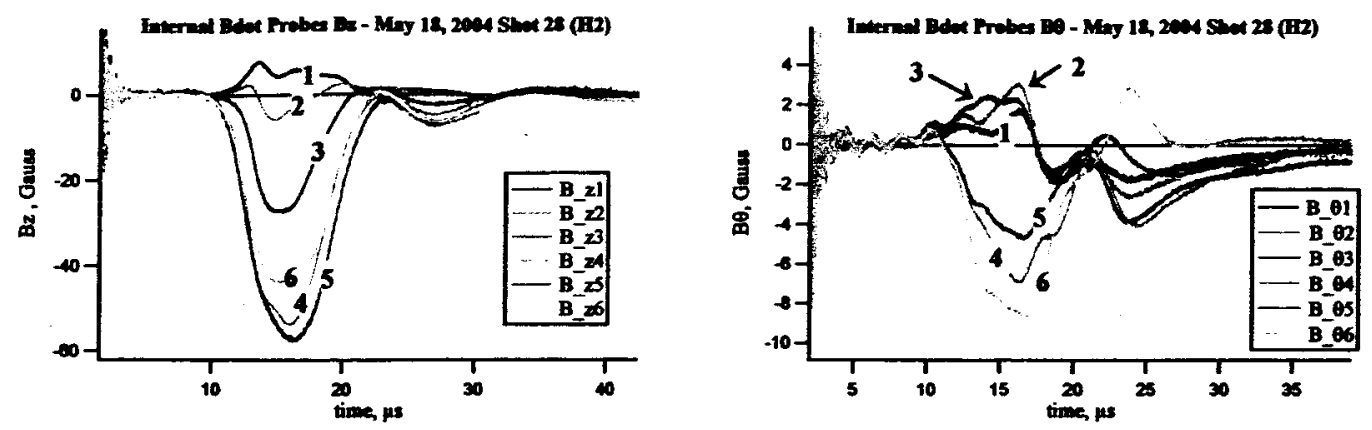

Figure 11. Plasmoid Magnetic Fields, May 18, 2004, Shot 28, 38.6 psig Hydrogen at $2400 \mu$ s delay
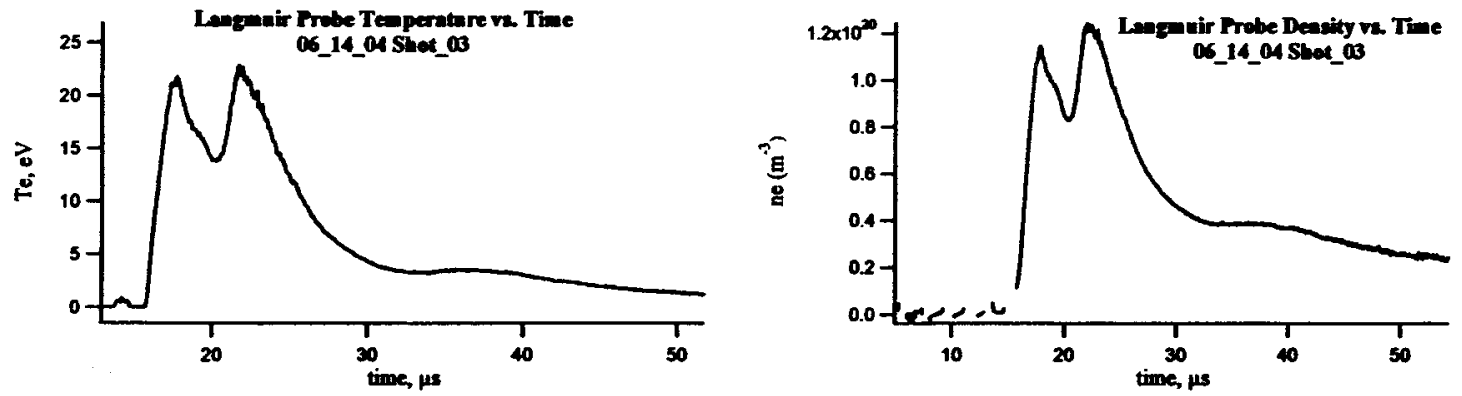

Figure 12. Plasmoid Temperature and Density, June 14, 2004, Shot 3, 38.7 psig Hydrogen at $2400 \mu$ s delay

\section{v. Discussion}

The magnetic field plots obtained from the B-dot probe array are consistent with plasmoid magnetic field configurations,' so PTX appears to be producing plasmoids. In the tests presented, the peak electron temperature, electron number density, and velocity ratio were $7.6 \mathrm{eV}, 4.9 \times 10^{-19} \mathrm{~m}^{-3}$, and 3.2 for argon, and $23 \mathrm{eV}$, $1.2 \times 10^{-20} \mathrm{~m}^{-3}$, and 0.4 for hydrogen. Hydrogen produced higher temperatures and densities, and lower $v / c_{\mathrm{m}}$, than argon, as expected with a lighter gas. The two peaks in the hydrogen temperature and density plots may indicate that two plasmoids are being formed during a single shot. In addition, some of the azimuthal B-dot probes show magnetic fields changing direction twice, which may indicate that two plasmoids are being formed in successive cycles of the ringing discharge. A large volume of PTX data, which is sufficiently reproducible for similar operating conditions, has been collected and is being analyzed.

\section{Acknowledgments}

Syri Koelfgen is funded by the NASA/MSFC Graduate Student Researchers Program (GSRP) fellowship. This research was conducted in the NASAMMSC Propulsion Research Center (TD40) laboratories. The authors would like to thank Jeff Richeson, Tommy Reid, and Doug Galloway of Mainthia for their technical support of PTX.

\section{References}

'Tuszewski, M., "Field Reversed Configurations - Review Paper," Nuclear Fusion, Vol. 28, No. 11, 1988, pp. $2033-2092$.

${ }^{2}$ Hoffiman, A. L., et al., "Inductive Field-Reversed Configuration Accelerator for Tokamak Fueling" Fusion Technology, Vol. 36, Sept. 1999.

${ }^{3}$ Miley, G. H., “Compact tori for alternate fuel fusion," Nuclear Instruments and Methods in Physics Research, Vol. 207, No. 1-2, 15 March 1983, pp. 111-120.

${ }^{4}$ Kawai, K., Pietrzyk, Z. A., and Hunter, H. T., "Generation of poloidally rotating spheromaks by the conical theta pinch," Phys. Fluids, Vol. 30, No. 8, Aug. 1987, pp. 2561-2568.

'Jahn, R. G., Physics of Electric Propulsion, McGraw-Hill, 1968. 
${ }^{6}$ Bourque, R. F., and Parks, P. B., "Time-Dependent Analysis of the Pulsed Plasmoid Electric Thruster," ALAANASAOAI Conference on Advanced SEI Technologies, AIAA Paper 91-3614, Cleveland, OH, 4-6 Sept. 1991.

${ }^{7}$ Slough, J., "Propagating Magnetic Wave Plasma Accelerator (PMWAC) for Deep Space Exploration," STTR Phase-1 Final Report, 2000.

${ }^{8}$ Koelfgen, S. J., Eskridge, R, Hawk, C. W., Lee, M. H. Martin, A., and Smith, J. W., "A Plasmoid Thruster for Space Propulsion" 39" ALAA/ASMESAE/ASEE Joint Propulsion Conference \& Exhibit, AIAA Paper 2003-4992, Huntsville, AL, 2023 July 2003.

'Myers, R M., et al., "Cathode Phenomena in a Low-Power Magnetoplasmadynamic Thruster," Journal of Propulsion and Power, Vol. 7, No. 5, Sept.-Oct. 1991, pp. 760-766.

${ }^{10}$ Hutchinson, I. H., Principles of Plasma Diagnastics, Cambridge University Press, 1987.

${ }^{1}$ Huddlestone, R. H., and Leonard, S. L., Plasma Diagnostic Techniques, Academic Press, 1965.

${ }^{12}$ Burton, R. L., DelMedico, S. G., and Andrews, J. C., "Application of a Quadruple Probe Technique to MPD Thruster Plume Measurements," Jowrnal of Propulsion and Power, Vol. 9, No. 5, Sept.-Oct. 1995, pp. 771-777.

${ }^{13}$ Bufton, S. A., "Exit Plane Plasma Measurements of a Low-Power Hydrazine Arcjet," Ph.D. Dissertation, Univ. of Illinois, Urbana-Champaign, 1996.

${ }^{14}$ Tilley, D. L., Kelly, A. J., and Jahn, R. G., "The Application of the Triple Probe Method to MPD Thruster Plumes," 21" AIAA/DGLRJJSASS International Electric Propulsion Conference, AIAA Paper 90-2667, Orlando, FL, $18-20$ July 1990.

${ }^{15}$ Chen, S. L., and Sekiguchi, T., "Instantaneous Direct-Display System of Plasma Parameters by Means of Triple Probe," Journal of Applied Physics, Vol. 36, No. 8, Aug. 1965, pp. 2363-2375.

${ }^{16} \mathrm{Kanal}$, M., "Theory of Current Collection of Moving Cylindrical Probes," Journal of Applied Physics, Vol. 35, No. 6, June 1964 , pp. 1697-1703.

17Johnson, B. H., and Murphree, D. L., "Plasma Velocity Determination by Electrostatic Probes," ALAA Journal, Vol. 7, Oct. 1969, pp. 2028-2030. 